\title{
On the Number of Balanced Words of Given Length and Height over a Two-Letter Alphabet
}

\author{
Nicolas Bédaride ${ }^{1}$ and Eric Domenjoud ${ }^{2}$ and Damien Jamet ${ }^{2}$ and Jean- \\ Luc Rémy ${ }^{2}$ \\ ${ }^{1}$ Université Aix-Marseille III, Avenue de l'Escadrille Normandie-Niémen, 13397 Marseille Cedex 20, France, \\ nicolas.bedaride@univ-cezanne. fr \\ ${ }^{2}$ Loria - Université Nancy 1 - CNRS, Campus Scientifique, BP 239, 54506 Vandouvre-lès-Nancy, France, \\ \{eric.domenjoud, damien.jamet, jean-luc.remy\}eloria.fr
}

received February 22, 2008, revised February 22, 2008, accepted tomorrow.

We exhibit a recurrence on the number of discrete line segments joining two integer points using an encoding of such segments as balanced words of given length and height over the two-letter alphabet $\{0,1\}$. We give generating functions and study the asymptotic behaviour. As a particular case, we focus on the symmetrical discrete segments which are encoded by balanced palindromes.

Keywords: keywords : to be done !

\section{Introduction}

The first investigations on discrete lines are dated back to E.B. Christoffel (Chr75), A. Markoff (Mar82) and more recently to G.A. Hedlund and H. Morse (MH40) who introduced the terminology of sturmian sequences, for the ones defined on a two-letter alphabet and coding lines with irrational slope. These works gave the first theoretical framework for discrete lines. A sequence $u \in\{0,1\}^{\mathbb{N}}$ is sturmian if and only if it is balanced and non-eventually periodic. From the 70's, H. Freeman (Fre74), A. Rosenfeld (Ros74) and S. Hung (Hun85) extended these investigations to lines with rational slope and studied discrete segments.

In (Rev91), J.-P. Reveillès defined arithmetic discrete lines as sets of integer points between two parallel Euclidean lines. More precisely, given a vector $\mathbf{v} \in \mathbb{R}^{2}$ and two real numbers $\mu \in \mathbb{R}$ and $w \in \mathbb{R}$, the arithmetic discrete line $\mathbf{D}(\mathbf{v}, \mu, w)$ with normal vector $\mathbf{v} \in$, shift $\mu$ and thickness $w$ is the subset of $\mathbb{Z}^{2}$ defined by $\mathbf{D}(\mathbf{v}, \mu, w)=\left\{\mathbf{x} \in \mathbb{Z}^{2},-\frac{w}{2} \leq\langle\mathbf{v}, \mathbf{x}\rangle+\mu<\frac{w}{2}\right\}$, where $\langle\mathbf{x}, \mathbf{y}\rangle=x_{1} y_{1}+x_{2} y_{2}$ denotes the usual scalar product in the canonical basis $\left\{\mathbf{e}_{1}, \mathbf{e}_{2}\right\}$ of $\mathbb{R}^{2}$. Moreover, if $w=\|\mathbf{v}\|_{\infty}$ (resp. $w=\|\mathbf{v}\|_{1}$ ), then the arithmetic discrete line $\mathbf{D}(\mathbf{v}, \mu, w)$ is called naive (resp. standard).

There exists a direct relation between naive (resp. standard) discrete arithmetic lines and sturmian sequences. Indeed, given a sturmian sequence $u \in\{0,1\}^{\mathbb{N}}$, if one associates the letters 0 and 1 with subm. to DMTCS (c) by the authors Discrete Mathematics and Theoretical Computer Science (DMTCS), Nancy, France 


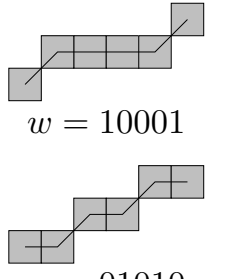

$w=01010$

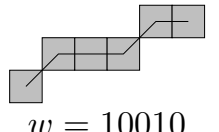

$w=10010$

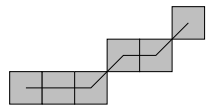

$w=00101$
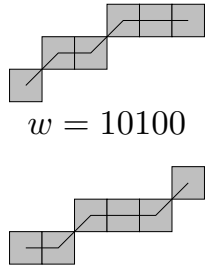

$w=01001$

Figure 1: There exist six discrete segments of length 5 and height 2.

a shifting along the vector $\mathbf{e}_{1}$ and $\mathbf{e}_{2}$ (resp. the vectors $\mathbf{e}_{1}$ and $\mathbf{e}_{1}+\mathbf{e}_{2}$ ) respectively, then, the vertices of the obtained broken line are the ones of a naive arithmetic discrete line (resp. a standard arithmetic discrete line) with the same slope (see Figure 1).

Let $s: \mathbb{N} \longmapsto \mathbb{N}$ be the map defined by:

$$
\begin{aligned}
& s: \mathbb{N} \longrightarrow \mathbb{N} \\
& L \quad \mapsto \quad \#\left\{w \in\{0,1\}^{L}, w \text { is balanced }\right\},
\end{aligned}
$$

where \#E denotes the cardinal of the set $E$. In other words, given $L \in \mathbb{N}, s(L)$ is the number of balanced words of length $L$, or equivalently, the number of discrete segments of slope $\alpha \in[0,1]$ of length $L$. In (BL88), it is proved that

$$
s(L)=1+\sum_{i=1}^{L}(L-i+1) \varphi(i),
$$

where $\varphi$ is Euler's totient function, that is, $\varphi(n)$ is the number of positive integers smaller that $n$ and coprime with $n$. Alternative proofs of this result can be found in (Mig91; BP93; CHT02).

In (dLdL05), the De Lucas investigated the number $p(L)$ of balanced palindrome words of length $L \in \mathbb{N}$, that is the balanced words coding a symmetrical discrete segments of length $L$. They proved

$$
p(L)=1+\sum_{i=0}^{\lceil L / 2\rceil-1} \varphi(L-2 i) .
$$

In the present work, we investigate the following question. Given two integer points of $\mathbb{Z}^{2}$ (also called pixels in the discrete geometry literature (CM91)), how many naive discrete segments link these points (see Figure 11? In other words, given $L \in \mathbb{N}$ and $h \in \mathbb{N}$, how much is $s(L, h)=\#\left\{w \in\{0,1\}^{L},|w|_{1}=\right.$ $h$ and $w$ balanced $\}$ ? We exhibit a recurrence relation on $s(L, h)$ and generating functions and we study the asymptotic behaviour of the maps $s$. After this, we focus on the number $p(L, h)$ of balanced palindromes of given length and height for which we also exhibit a recurrence relation and a generating function.

\section{Basic notions and notation}

Let $\{0,1\}^{*}$ and $\{0,1\}^{\mathbb{N}}$ be the set of respectively finite and infinite words on the alphabet $\{0,1\}$. We denote the empty word by $\epsilon$. For any word $w \in\{0,1\}^{*},|w|$ denotes the length of $w$, and $|w|_{0}$ and $|w|_{1}$ denote respectively the number of 0's and 1's in $w .|w|_{1}$ is also called the height of $w$. A (finite or infinite) 
word $w$ is balanced if and only if for any finite subwords $u$ and $v$ of $w$ such that $|u|=|v|$, we have $\left.|| u\right|_{0}-|v|_{0} \mid \leq 1$. The letter $x$ (0 or 1$)$ is isolated in a word $w$ if $w$ does not contain the subword $x x$. Observe that in any balanced word, at least one letter is isolated. A (finite or infinite) word $w$ is of type 0 (resp. type 1) if the letter 1 (resp. the letter 0) is isolated in $w$.

We denote by $\mathbb{S}$ the set of finite balanced words and by $\mathbb{S}^{0}$ (resp. $\mathbb{S}^{1}$ ) the set of finite balanced words of type 0 (resp. 1).

Let $L, h \in \mathbb{N}$ and $\alpha, \beta \in\{0,1\}^{*}$. We denote by $\mathbb{S}_{\alpha, \beta}(L, h)$ the set of elements of $\mathbb{S}$ of length $L$ and height $h$, of which $\alpha$ is a prefix and $\beta$ is a suffix. Note that $\alpha$ and $\beta$ may overlap. For short, we usually write $\mathbb{S}(L, h)$ instead of $\mathbb{S}_{\epsilon, \epsilon}(L, h)$. Observe that $\mathbb{S}(L, h)$ is the set of finite balanced words which encode the discrete segments between $(0,0)$ and $(L, h)$.

We define the function $s(L, h)$ on $\mathbb{Z}^{2}$ by:

$$
s(L, h)= \begin{cases}\# \mathbb{S}(L, h \bmod L) & \text { if } L>0 \\ 1 & \text { if } L=0 \text { and } h=0 \\ 0 & \text { if } L<0 \text { or } L=0 \text { and } h \neq 0\end{cases}
$$

Observe that for $0 \leq h \leq L$, since $\# \mathbb{S}(L, L)=\# \mathbb{S}(L, 0)$, one has $s(L, h)=\# \mathbb{S}(L, h)$ and, for all $L, h, k \in \mathbb{Z}, s(L, h)=s(L, h+k L)$.

For $0 \leq h \leq L$ and $\alpha, \beta \in\{0,1\}^{*}$ we denote by $s_{\alpha, \beta}(L, h)$ the cardinal of $\mathbb{S}_{\alpha, \beta}(L, h)$. Notice that $s_{\alpha, \beta}(L, h)=s_{\bar{\alpha}, \bar{\beta}}(L, L-h)$, where $\bar{w}$ is the word obtained by replacing the 0's with 1's and the 1's with 0 's in $w$.

\section{General case}

\subsection{Main theorem}

In the present section, we prove the following result:

Theorem 1 For all $L, h \in \mathbb{N}$ satisfying $0 \leq h \leq L / 2$, one has:

$$
s(L, h)=s(L-h-1, h)+s(L-h, h)-s(L-2 h-1, h)+s(h-1, L-2)+s(h-1, L-1) .
$$

In order to prove Theorem 1, let us now introduce some technical definitions and lemmas. Let $\varphi_{0}$ be the morphism defined on $\{0,1\}^{*}$ and $\{0,1\}^{\mathbb{N}}$ by:

$$
\varphi_{0}: \begin{array}{ccc}
0 & \mapsto 0 \\
1 & \mapsto 01
\end{array}
$$

Let us recall that $\varphi_{0}$ is a sturmian morphism, that is, for any sturmian sequence $u$, the sequence $\varphi_{0}(u)$ is sturmian (Par97). Moreover:

Lemma $2(\underline{(\mathbf{L o t 0 2})})$ Let $w \in\{0,1\}^{\mathbb{N}}$.

1. If $0 w$ is sturmian of type 0 , then there exists a unique sturmian sequence $u$ satisfying $\varphi_{0}(u)=0 w$.

2. $w$ is sturmian if and only if so is $\varphi_{0}(w)$. 
Since every balanced word is a factor of a sturmian word, we directly deduce:

Corollary 3 If a finite word $w \in\{0,1\}^{*}$ is balanced then so is $\varphi_{0}(w)$.

Definition 1 (0-erasing map) Let $\theta_{0}:\{0,1\}^{*} \rightarrow\{0,1\}^{*}$ be the map defined by induction as follows:

$$
\begin{aligned}
& \theta_{0}(\epsilon) \quad=\epsilon \\
& \theta_{0}\left(0^{\alpha+1}\right)=0^{\alpha} \quad \text { for } \alpha \geq 0 \\
& \theta_{0}(1 v)=1 \theta_{0}(v) \\
& \theta_{0}\left(0^{\alpha+1} 1 v\right)=0^{\alpha} 1 \theta_{0}(v) \quad \text { for } \alpha \geq 0
\end{aligned}
$$

Roughly speaking, $\theta_{0}$ erases a 0 in each maximal range of 0 in a given word. In some sense, $\theta_{0}$ is the inverse of $\varphi_{0}$. Let us now prove some key properties of $\theta_{0}$ :

Lemma 4 Consider the set $\mathbb{S}_{0,1}^{0}=\left\{u, u \in \mathbb{S}^{0}, \exists w \in\{0,1\}^{*}, u=0 w 1\right\}$ of words in $\mathbb{S}^{0}$ of the form $0 w 1$ with $w \in\{0,1\}^{*}$. Then

- The maps $\varphi_{0}$ and $\theta_{0}$ are bijective and in correspondence on this set.

- Moreover we have $\theta_{0}\left(\varphi_{0}(w 1)\right)=w 1$ for all $w$.

Proof: By induction on $|w|_{1}$.

1. If $|w|_{1}=0$ then $w=0^{\alpha}$ for some $\alpha \geq 0$ and we have $\varphi_{0}\left(\theta_{0}\left(00^{\alpha} 1\right)\right)=\varphi_{0}\left(0^{\alpha} 1\right)=0^{\alpha+1} 1=0 w 1$.

2. Assume $|w|_{1} \geq 1$ and the result holds for all $u$ such that $|u|_{1}<|w|_{1}$. We have $w=0^{\alpha} 1 w^{\prime}$ for some $\alpha \geq 0$. By assumption, the letter 1 is isolated in $0 w 1$, so that $w^{\prime} \neq \epsilon$ and $w^{\prime}$ starts with the letter 0 . Hence,

$$
\varphi_{0}\left(\theta_{0}(0 w 1)\right)=\varphi_{0}\left(\theta_{0}\left(0^{\alpha+1} 1 w^{\prime} 1\right)\right)=\varphi_{0}\left(0^{\alpha} 1 \theta_{0}\left(w^{\prime} 1\right)\right)=0^{\alpha} 01 \varphi_{0}\left(\theta_{0}\left(w^{\prime} 1\right)\right) .
$$

By the induction hypothesis, we obtain

$$
\varphi_{0}\left(\theta_{0}(0 w 1)\right)=0^{\alpha+1} 1 w^{\prime} 1=0 w 1 .
$$

Example 1 We have by straightforward computations: $\varphi_{0}\left(\theta_{0}(11)\right)=0101$. Thus the maps are not in correspondence everywhere.

Lemma 5 Let $w \in\{0,1\}^{*}$. If $w$ is balanced then so is $\theta_{0}(w)$.

\section{Proof:}

1. If $w$ is of type 1 (i.e. the letter 0 is isolated in $w$ ), then we check that $\theta_{0}(w)=1^{\alpha}$ for some integer $\alpha$. Hence it is balanced. 
2. Assume now that $w \in \mathbb{S}_{0}$.

- There exist $\alpha \in\{0,1\}, \beta \in \mathbb{N}$ and a sturmian sequence $u$ of type 0 such that the sequence $0^{\alpha} w 0^{\beta} 1 u$ is sturmian and starts with the letter 0 . Notice that $u$ starts with the letter 0 too.

- By point 1 of Lemma 2, there exists a sturmian sequence $u^{\prime}$ such that $u=\varphi_{0}\left(u^{\prime}\right)$.

- We have

$$
\begin{aligned}
\varphi_{0}\left(\theta_{0}\left(0^{\alpha} w 0^{\beta} 1\right) u^{\prime}\right) & =\varphi_{0}\left(\theta_{0}\left(0^{\alpha} w 0^{\beta} 1\right)\right) \varphi_{0}\left(u^{\prime}\right) & & \text { since } \varphi_{0} \text { is a morphism } \\
& =0^{\alpha} w 0^{\beta} 1 u & & \text { by Lemma } 4 .
\end{aligned}
$$

and by point 2 of Lemma $2 \theta_{0}\left(0^{\alpha} w 0^{\beta} 1\right) u^{\prime}$ is sturmian because $0^{\alpha} w 0^{\beta} 1 u$ is sturmian. Hence $\theta_{0}\left(0^{\alpha} w 0^{\beta} 1\right)$ is balanced as a factor of a balanced word. Finally, we prove by induction on $|w|_{1}$ that $\theta_{0}\left(0^{\alpha} w 0^{\beta} 1\right)=0^{\alpha^{\prime}} \theta_{0}(w) 0^{\beta^{\prime}} 1$ for some integers $\alpha^{\prime}$ and $\beta^{\prime}$, so that $\theta_{0}(w)$ is balanced as a factor of a balanced word.

$\square$ The last technical property of $\theta_{0}$ we need is:

\section{Lemma 6}

1. If $L \geq 2 h+1$ then $\theta_{0}$ is a bijection from $\mathbb{S}_{0,0}(L, h)$ to $\mathbb{S}_{\epsilon, \epsilon}(L-(h+1), h)$

2. If $L \geq 2 h$ then $\theta_{0}$ is a bijection from $\mathbb{S}_{0,1}(L, h)$ to $\mathbb{S}_{\epsilon, 1}(L-h, h)$ and from $\mathbb{S}_{1,0}(L, h)$ to $\mathbb{S}_{1, \epsilon}(L-$ $h, h)$.

3. If $L \geq 2 h-1$ then $\theta_{0}$ is a bijection from $\mathbb{S}_{1,1}(L, h)$ to $\mathbb{S}_{1,1}(L-(h-1), h)$

Proof: If $h=0$ and $L \neq 0, \mathbb{S}_{0,0}(L, h)=\left\{0^{L}\right\}$ and $\mathbb{S}_{\epsilon, \epsilon}(L-(h+1), h)=\left\{0^{L-1}\right\}=\left\{\theta_{0}\left(0^{L}\right)\right\}$. All others sets are empty so that the result obviously holds. In the rest of the proof, we assume $h \geq 1$. We prove the result for $\mathbb{S}_{0,1}(L, h)$. The proof of other cases is similar and left to the reader.

Notice first that $\mathbb{S}_{0,1}(L, h) \subset \mathbb{S}^{0}$ iff $L \geq 2 h$. Indeed, if $L=2 h$, then $\mathbb{S}_{0,1}(L, h)=\left\{(01)^{h}\right\} \subset \mathbb{S}^{0}$. Now, if $L>2 h$, by the pigeonhole principle, any $w \in \mathbb{S}_{0,1}(L, h)$ must contain the subword 00. Since $w$ is balanced, it cannot contain the subword 11 hence the letter 1 is isolated. Conversely, if the letter 1 is isolated in $w$, then $w$ must contain at least $h 0$ 's, hence $L \geq 2 h$.

- $\theta_{0}\left(\mathbb{S}_{0,1}(L, h)\right) \subset \mathbb{S}_{\epsilon, 1}(L-h, h)$

Let $w \in \mathbb{S}_{0,1}(L, h)$. By an easy induction on $h$, we show that $\left|\theta_{0}(w)\right|=L-h$ and $\left|\theta_{0}(w)\right|_{1}=$ $|w|_{1}=h$. Furthermore, from Lemma 5, $\theta_{0}(w)$ is balanced so that $\theta_{0}(w) \in \mathbb{S}(L-h, h)$. Now from the definition of $\theta_{0}$, if 1 is a suffix of $w$, then it is also a suffix of $\theta_{0}(w)$ so that $\theta_{0}(w) \in$ $\mathbb{S}_{\epsilon, 1}(L-h, h)$.

- $\theta_{0}: \mathbb{S}_{0,1}(L, h) \rightarrow \mathbb{S}_{\epsilon, 1}(L-h, h)$ is injective.

Let $u, v \in \mathbb{S}_{0,1}(L, h)$. We have $u=0^{\alpha+1} 1 u^{\prime}, v=0^{\beta+1} 1 v^{\prime}$ and

$$
\theta_{0}(u)=\theta_{0}(v) \Leftrightarrow 0^{\alpha} 1 \theta_{0}\left(u^{\prime}\right)=0^{\beta} 1 \theta_{0}\left(v^{\prime}\right) \Leftrightarrow \alpha=\beta \wedge \theta_{0}\left(u^{\prime}\right)=\theta_{0}\left(v^{\prime}\right)
$$

Now, either $h=1$ and $u^{\prime}=v^{\prime}=\epsilon$ so that $u=v$ or $h>1$ and $u^{\prime}, v^{\prime} \in \mathbb{S}_{0,1}(L-\alpha-1, h-1)$. We get the result by induction on $h$. 
- $\theta_{0}: \mathbb{S}_{0,1}(L, h) \rightarrow \mathbb{S}_{\epsilon, 1}(L-h, h)$ is surjective.

Let $w \in \mathbb{S}_{\epsilon, 1}(L-h, h)$. We have $w^{\prime}=\varphi_{0}(w) \in \mathbb{S}_{0,1}(L, h)$. Indeed, $w^{\prime}$ is balanced because $w$ is, $\left|w^{\prime}\right|=|w|_{0}+2|w|_{1}=L-2 h+2 h=L$ and $\left|w^{\prime}\right|_{1}=|w|_{1}=h$ so that $w^{\prime} \in \mathbb{S}(L, h)$. Since 1 is a suffix of $w$, it is also a suffix of $w^{\prime}$ and from Lemma $4, \theta_{0}\left(w^{\prime}\right)=w$.

As already said, the proof of other cases is similar. To prove that $\theta_{0}: \mathbb{S}_{0,0}(L, h) \rightarrow \mathbb{S}_{\epsilon, \epsilon}(L-(h+1), h)$ is surjective, we consider for each $w \in \mathbb{S}_{\epsilon, \epsilon}(L-(h+1), h), w^{\prime}=\varphi_{0}(w) 0$. Then we have $w^{\prime} \in \mathbb{S}_{0,0}(L, h)$ and $\theta_{0}\left(w^{\prime}\right)=w$. For $\theta_{0}: \mathbb{S}_{1,0}(L, h) \rightarrow \mathbb{S}_{1, \epsilon}(L-h, h)$, we consider for each $w \in \mathbb{S}_{1, \epsilon}(L-h, h)$, $w^{\prime}=w^{\prime \prime} 0$ where $\varphi_{0}(w)=0 w^{\prime \prime}$. Finally, for $\theta_{0}: \mathbb{S}_{1,1}(L, h) \rightarrow \mathbb{S}_{1,1}(L-(h-1), h)$, we consider for each $w \in \mathbb{S}_{1,1}(L-(h-1), h), w^{\prime}=w^{\prime \prime}$ where $\varphi_{0}(w)=0 w^{\prime \prime}$.

Corollary 7 For all $L, h$ such that $2 \leq h \leq L, s_{1,1}(L, h)=s_{1,1}(h+(L-h) \bmod (h-1), h)$.

Proof: Follows from case 3 by induction on $q=\left\lfloor\frac{L-h}{h-1}\right\rfloor$.

Lemma 8 For all $L, h$ such that $0 \leq h \leq L, s_{0,0}(L, h)=s(L-h-1, h)$ and $s_{1,1}(L, h)=s(h-1, L-1)$.

Proof: We distinguish several cases.

- If $2 h<L$, the result is an immediate consequence of case 1 of Lemma 6 .

- If $h+1<L \leq 2 h$ (which implies $h \geq 2$ ), we have

$$
\begin{aligned}
s_{0,0}(L, h) & =s_{1,1}(L, L-h) \\
& =s_{1,1}(L-h+h \bmod (L-h-1), L-h) \\
& =s_{0,0}(L-h+h \bmod (L-h-1), h \bmod (L-h-1)) \\
& =s(L-h-1, h \bmod (L-h-1)) \\
& =s(L-h-1, h)
\end{aligned}
$$

by exchanging 0 's and 1 's by Corollary 7 by exchanging 0 's and 1 's by case 1 of Lemma 6 by definition of $s(L, h)$

- If $L=h+1$ we have $L-h-1=0$. Either $h=0$ and $L=1$ in which case we have $s_{0,0}(1,0)=\#\{0\}=1=\#\{\epsilon\}=s(0,0)$, or $h>0$ and we have $s_{0,0}(L, h)=0=s(0, h)$.

- If $L=h$ we have $s_{0,0}(h, h)=0=s(-1, h)$.

Since $s_{1,1}(L, h)=s_{0,0}(L, L-h)$, we immediately obtain $s_{1,1}(L, h)=s(h-1, L-h)$. Moreover $s(L, h)=s(L, h \bmod L)$, so that $s_{1,1}(L, h)=s(h-1, L-1)$.

We are now ready to prove the main theorem. Proof of Theorem 1; One easily checks that the property holds for $L=0$. If $L>0$, then the following disjoint union holds:

$$
\mathbb{S}(L, h)=\mathbb{S}_{0,0}(L, h) \uplus \mathbb{S}_{0,1}(L, h) \uplus \mathbb{S}_{1,0}(L, h) \uplus \mathbb{S}_{1,1}(L, h),
$$


and, consequently:

$$
s(L, h)=s_{0,0}(L, h)+s_{0,1}(L, h)+s_{1,0}(L, h)+s_{1,1}(L, h) .
$$

From Lemmas 6 and 8 , it follows that

$$
\begin{aligned}
s(L, h) & =s(L-h-1, h)+s_{\epsilon, 1}(L-h, h)+s_{1, \epsilon}(L-h, h)+s(h-1, L-1), \\
& =s(L-h-1, h)+s(L-h, h)+s_{1,1}(L-h, h)-s_{0,0}(L-h, h)+s(h-1, L-1), \\
& =s(L-h-1, h)+s(L-h, h)+s(h-1, L-2)-s(L-2 h-1, h)+s(h-1, L-1) .
\end{aligned}
$$

To summarize, we obtain the following recurrence for $s(L, h)$

Theorem 9 Let $L, h \in \mathbb{Z}$,

$$
s(L, h)= \begin{cases}0 & \text { if } L<0 \text { or }(L=0 \text { and } h \neq 0) \\ 1 & \text { if } L \geq 0 \text { and } h=0 \\ s(L, h \bmod L) & \text { if } L>0 \text { and }(h<0 \text { or } h>L) \\ s(L, L-h) & \text { if } L>0 \text { and } L / 2<h \leq L \\ s(L-h-1, h)+s(L-h, & \text { - }-s(L-2 h-1, h)+s(h-1, L-2) \\ & \text { if } L>0 \text { and } 0<h \leq L / 2\end{cases}
$$

Sample values of $s(L, h)$ are given in table 1 .

\begin{tabular}{r||rrrrrrrrrrr}
$L \backslash h$ & 0 & 1 & 2 & 3 & 4 & 5 & 6 & 7 & 8 & 9 & 10 \\
\hline 0 & 1 & & & & & & & & & & \\
1 & 1 & 1 & & & & & & & & & \\
2 & 1 & 2 & 1 & & & & & & & & \\
3 & 1 & 3 & 3 & 1 & & & & & & & \\
4 & 1 & 4 & 4 & 4 & 1 & & & & & & \\
5 & 1 & 5 & 6 & 6 & 5 & 1 & & & & & \\
6 & 1 & 6 & 8 & 6 & 8 & 6 & 1 & & & & \\
7 & 1 & 7 & 11 & 8 & 8 & 11 & 7 & 1 & & & \\
8 & 1 & 8 & 13 & 12 & 8 & 12 & 13 & 8 & 1 & & \\
9 & 1 & 9 & 17 & 13 & 12 & 12 & 13 & 17 & 9 & 1 & \\
10 & 1 & 10 & 20 & 16 & 16 & 10 & 16 & 16 & 20 & 10 & 1
\end{tabular}

Table 1: Sample values of $s(L, h)$ for $0 \leq h \leq L \leq 10$

\subsection{An explicit formula for $s(L, 2)$}

Using the recurrence formula of Theorem 1, we can deduce an explicit formula for some particular cases. For instance, one has: 
Proposition 10 Let $L \geq 0$ be an integer. Then, one has:

$$
s(L, 2)=\left\lfloor\frac{(L+1)^{2}+2}{6}\right\rfloor .
$$

Proof: By induction on $L$. One checks that the result holds for $L \in\{0,1,2,3,4\}$. Assume $L \geq 5$ and the result holds for all nonnegative integers smaller than $L$. From Theorem 1 , one deduces $s(L, 2)-s(L-$ $3,2)=s(L-2,2)-s(L-5,2)+2$.

For all $L \geq 3$, let $u_{L}=s(L, 2)-s(L-3,2)$. Then, $u_{L+2}=u_{L}+2$ and one obtains $u_{L}=$ $L-1+(L \bmod 2)$. By induction, it follows:

$$
s(L, 2)=\left\lfloor\frac{(L-2)^{2}+2}{6}\right\rfloor+L-1+(L \bmod 2)=\left\lfloor\frac{L^{2}+2 L}{6}\right\rfloor+(L \bmod 2)
$$

Finally, it suffices to check that:

$$
\left\lfloor\frac{L^{2}+2 L}{6}+\frac{1}{2}\right\rfloor=\left\lfloor\frac{L^{2}+2 L}{6}\right\rfloor+(L \bmod 2)
$$

By considering the remainder of $L$ modulo 6 , we obtain that the fractional part of $\frac{L^{2}+2 L}{6}$ is strictly less than $\frac{1}{2}$ if and only if $L$ is even. The result follows.

\subsection{Generating functions of $s(L, h)$}

A classical way to obtain an explicit formula of a given function consists in computing its generating function. In this section, we exhibit for each $h \geq 0$ the generating function $\mathcal{S}_{h}(X)$ of $s(L, h)$, namely $\mathcal{S}_{h}(X)=\sum_{L \geq 0} s(L, h) X^{L}$. Let us recall that, in that case, $s(L, h)=\frac{\mathcal{S}_{h}^{(L)}(0)}{L !}$.

Theorem 11 One has: $\mathcal{S}_{0}(X)=\frac{1}{1-X}, \mathcal{S}_{1}(X)=\frac{X}{1-X^{2}}$ and for all $h \geq 2$,

$$
\begin{array}{r}
\mathcal{S}_{h}(X)=\left[\left(1-X^{h-1}\right)\left(\sum_{L=0}^{2 h-1} s(L, h) X^{L}-\sum_{L=0}^{h-1} s(L, h) X^{L+h}-\sum_{L=0}^{h-2} s(L, h) X^{L+h+1}-X^{2 h-1}\right)\right. \\
\left.+(1+X) \sum_{r=0}^{h-2} s(h-1, r) X^{r+2 h-1}\right] /\left(\left(1-X^{h-1}\right)\left(1-X^{h}\right)\left(1-X^{h+1}\right)\right)
\end{array}
$$

Proof: We have immediately

$$
\mathcal{S}_{0}(X)=\sum_{L \geq 0} X^{L}=\frac{1}{1-X} \quad \text { and } \quad \mathcal{S}_{1}(X)=\sum_{L \geq 0} L X^{L}=\frac{X}{(1-X)^{2}}
$$


and from the previous recurrence, for $h \geq 2$, we get: 


$$
\begin{aligned}
\mathcal{S}_{h}(X) & =\sum_{L \geq 0} s(L, h) X^{L} \\
& =\sum_{L=0}^{2 h-1} s(L, h) X^{L}+\sum_{L \geq 2 h} s(L, h) X^{L} \\
& =\sum_{L=0}^{2 h-1} s(L, h) X^{L}+\sum_{L \geq 2 h}\left(\begin{array}{r}
(L-h-1, h)+s(L-h, h)-s(L-2 h-1, h) \\
+s(h-1, L-1)+s(h-1, L-2)) X^{L}
\end{array}\right.
\end{aligned}
$$

by using the recurrence of Th. 1

$$
\begin{aligned}
= & \sum_{L=0}^{2 h-1} s(L, h) X^{L}+\sum_{L \geq 2 h} s(L-h-1, h) X^{L}+\sum_{L \geq 2 h} s(L-h, h) X^{L} \\
& -\sum_{L \geq 2 h} s(L-2 h-1, h) X^{L}+\sum_{L \geq 2 h}(s(h-1, L-1)+s(h-1, L-2)) X^{L} \\
= & \sum_{L=0}^{2 h-1} s(L, h) X^{L}+X^{h+1} \sum_{L \geq h-1} s(L, h) X^{L}+X^{h} \sum_{L \geq h} s(L, h) X^{L} \\
& \quad-X^{2 h+1} \sum_{L \geq-1} s(L, h) X^{L}+\sum_{L \geq 2 h}(s(h-1, L-1)+s(h-1, L-2)) X^{L} \\
= & \sum_{L=0}^{2 h-1} s(L, h) X^{L}+X^{h+1}\left(\mathcal{S}_{h}(X)-\sum_{L=0}^{h-2} s(L, h) X^{L}\right)+X^{h}\left(\mathcal{S}_{h}(X)-\sum_{L=0}^{h-1} s(L, h) X^{L}\right) \\
& \quad-X^{2 h+1} \mathcal{S}_{h}(X)+\left(X+X^{2}\right) \sum_{L \geq 2 h-2} s(h-1, L) X^{L}-s(h-1,0) X^{2 h-1} \\
= & \quad\left(X^{h}+X^{h+1}-X^{2 h+1}\right) \mathcal{S}_{h}(X)+\sum_{L=0}^{2 h-1} s(L, h) X^{L}-X^{h+1} \sum_{L=0}^{h-2} s(L, h) X^{L}-X^{h} \sum_{L=0}^{h-1} s(L, h) X^{L} \\
& \quad\left(X+X^{2}\right) \sum_{q \geq 2}^{h-2} \sum_{r=0}^{h} s(h-1, r) X^{q(h-1)+r}-X^{2 h-1}
\end{aligned}
$$

by setting $L=q \times(h-1)+r$

$$
\begin{aligned}
& =\left(X^{h}+X^{h+1}-X^{2 h+1}\right) \mathcal{S}_{h}(X)+\sum_{L=0}^{2 h-1} s(L, h) X^{L}-X^{h+1} \sum_{L=0}^{h-2} s(L, h) X^{L}-X^{h} \sum_{L=0}^{h-1} s(L, h) X^{L} \\
& \quad+\left(X+X^{2}\right) \sum_{q \geq 2} X^{q(h-1)} \sum_{r=0}^{h-2} s(h-1, r) X^{r}-X^{2 h-1} \\
& =\left(X^{h}+X^{h+1}-X^{2 h+1}\right) \mathcal{S}_{h}(X)+\sum_{L=0}^{2 h-1} s(L, h) X^{L}-X^{h+1} \sum_{L=0}^{h-2} s(L, h) X^{L}-X^{h} \sum_{L=0}^{h-1} s(L, h) X^{L} \\
& \quad+(1+X) \frac{X^{2 h-1}}{1-X^{h-1}} \sum_{r=0}^{h-2} s(h-1, r) X^{r}-X^{2 h-1}
\end{aligned}
$$




$$
\begin{aligned}
\mathcal{S}_{2}(X) & =\frac{X+X^{3}}{(1-X)\left(1-X^{2}\right)\left(1-X^{3}\right)} \\
\mathcal{S}_{3}(X) & =\frac{X+2 X^{2}+X^{4}+2 X^{5}}{\left(1-X^{2}\right)\left(1-X^{3}\right)\left(1-X^{4}\right)} \\
\mathcal{S}_{4}(X) & =\frac{X+X^{2}+3 X^{3}+3 X^{5}+3 X^{6}+3 X^{7}}{\left(1-X^{3}\right)\left(1-X^{4}\right)\left(1-X^{5}\right)} \\
\mathcal{S}_{5}(X) & =\frac{X+2 X^{2}+3 X^{3}+4 X^{4}+3 X^{6}+5 X^{7}+3 X^{8}+4 X^{9}+X^{12}}{\left(1-X^{4}\right)\left(1-X^{5}\right)\left(1-X^{6}\right)} \\
\mathcal{S}_{6}(X) & =\frac{X+X^{2}+X^{3}+4 X^{4}+5 X^{5}+5 X^{7}+10 X^{8}+7 X^{9}+6 X^{10}+5 X^{11}+X^{14}}{\left(1-X^{5}\right)\left(1-X^{6}\right)\left(1-X^{7}\right)}
\end{aligned}
$$

Table 2: Sample values of $\mathcal{S}_{h}(X)$

Finally, we get

$$
\begin{aligned}
& \mathcal{S}_{h}(X)= \frac{1}{\left(1-X^{h}\right)\left(1-X^{h+1}\right)}\left(\sum_{L=0}^{2 h-1} s(L, h) X^{L}-X^{h+1} \sum_{L=0}^{h-2} s(L, h) X^{L}-X^{h} \sum_{L=0}^{h-1} s(L, h) X^{L}\right. \\
&\left.+(1+X) \frac{X^{2 h-1}}{1-X^{h-1}} \sum_{r=0}^{h-2} s(h-1, r) X^{r}-X^{2 h-1}\right) \\
&= \\
&\left.+(1+X) \sum_{r=0}^{h-2} s(h-1, r) X^{r+2 h-1}\right] /\left(\left(1-X^{h-1}\right)\left(1-X^{h}\right)\left(1-X^{h+1}\right)\right) \\
&=\frac{\mathcal{F}_{h}(X)}{\left(1-\sum_{L=0}^{2 h-1} s(L, h) X^{L}-\sum_{L=0}^{h-1} s(L, h) X^{L+h}-\sum_{L=0}^{h-2} s(L, h) X^{L+h+1}-X^{2 h-1}\right)} \\
&
\end{aligned}
$$

where $\mathcal{F}_{h} \in \mathbb{Z}[X]$ and $\operatorname{deg}\left(\mathcal{F}_{h}\right) \leq 3 h-2$.

$\square$ Notice that the previous equality does provide a closed formula for $\mathcal{S}_{h}(X)$ although is still depends on $s(L, h)$ because each sum is finite. Sample values of $\mathcal{S}_{h}(X)$ are given in Table 2

\subsection{Asymptotic behaviour of $s(L, h)$}

Using the generating functions we just computed, we may deduce an expression of $s(L, h)$ which highlights its asymptotic behaviour when $L$ grows.

We prove the following theorem: 
Theorem 12 For all $h \geq 2$, there exist $u_{0}, \ldots, u_{h-2}, v_{0}, \ldots, v_{h-1}, w_{0}, \ldots, w_{h} \in \mathbb{Q}$ such that

$$
\forall L \geq 0, \quad s(L, h)=\alpha L^{2}+\beta L+u_{L \bmod (h-1)}+v_{L \bmod h}+w_{L \bmod (h+1)}
$$

with $\alpha=\frac{1}{h\left(h^{2}-1\right)} \sum_{i=1}^{h-1}(h-i) \varphi(i)$ and $\beta=\frac{1}{h(h+1)} \sum_{i=1}^{h} \varphi(i)$ where $\varphi$ is Euler's totient function.

Before proving this theorem, we need some preliminary results.

Lemma 13 For all $h \geq 2$, there exist $R, A, B, C \in \mathbb{Q}[X]$ such that $\operatorname{deg}(R)<3, \operatorname{deg}(A)<h-1$, $\operatorname{deg}(B)<h, \operatorname{deg}(C)<h+1$ and

$$
\mathcal{S}_{h}(X)=\frac{R(X)}{(1-X)^{3}}+\frac{A(X)}{1-X^{h-1}}+\frac{B(X)}{1-X^{h}}+\frac{C(X)}{1-X^{h+1}}
$$

Proof: We have

$$
\mathcal{S}_{h}(X)=\frac{\mathcal{F}_{h}(X)}{\left(1-X^{h-1}\right)\left(1-X^{h}\right)\left(1-X^{h+1}\right)}=\frac{\mathcal{F}_{h}(X)}{(1-X)^{3} \frac{1-X^{h-1}}{1-X} \frac{1-X^{h}}{1-X} \frac{1-X^{h+1}}{1-X}}
$$

where $\mathcal{F}_{h} \in \mathbb{Z}[X]$ and $\operatorname{deg}\left(\mathcal{F}_{h}\right) \leq 3 h-2$.

If $h$ is even, $(1-X)^{3}, \frac{1-X^{h-1}}{1-X}, \frac{1-X^{h}}{1-X}$ and $\frac{1-X^{h+1}}{1-X}$ are pairwise coprime so that

$$
\mathcal{S}_{h}=\frac{R(X)}{(1-X)^{3}}+\frac{A_{0}(X)}{\frac{1-X^{h-1}}{1-X}}+\frac{B_{0}(X)}{\frac{1-X^{h}}{1-X}}+\frac{C_{0}(X)}{\frac{1-X^{h+1}}{1-X}}
$$

for some $R, A_{0}, B_{0}, C_{0} \in \mathbb{Q}[X]$ with $\operatorname{deg}(R)<3, \operatorname{deg}\left(A_{0}\right)<h-2, \operatorname{deg}\left(B_{0}\right)<h-1$ and $\operatorname{deg}\left(C_{0}\right)<h$. The result follows with $A(X)=(1-X) A_{0}(X), B(X)=(1-X) B_{0}(X)$ and $C(X)=(1-X) C_{0}(X)$.

If $h$ is odd, $\frac{1-X^{h-1}}{1-X}$ and $\frac{1-X^{h+1}}{1-X}$ are dividable by $1+X$. But in this case, we notice that $\mathcal{F}_{h}(-1)=0$ so that $\mathcal{F}_{h}(X)$ is also dividable by $1+X$ and we may write

$$
\mathcal{S}_{h}(X)=\frac{\frac{\mathcal{F}_{h}(X)}{1+X}}{(1-X)^{3} \frac{1-X^{h-1}}{1-X} \frac{1-X^{h}}{1-X} \frac{1-X^{h+1}}{1-X^{2}}}
$$

where $(1-X)^{3}, \frac{1-X^{h-1}}{1-X}, \frac{1-X^{h}}{1-X}$ and $\frac{1-X^{h+1}}{1-X^{2}}$ are pairwise coprime. Thus we get

$$
\mathcal{S}_{h}=\frac{R(X)}{(1-X)^{3}}+\frac{A_{0}(X)}{\frac{1-X^{h-1}}{1-X}}+\frac{B_{0}(X)}{\frac{1-X^{h}}{1-X}}+\frac{C_{0}(X)}{\frac{1-X^{h+1}}{1-X^{2}}}
$$

for some $R, A_{0}, B_{0}, C_{0} \in \mathbb{Q}[X]$ with $\operatorname{deg}(R)<3$, $\operatorname{deg}\left(A_{0}\right)<h-2, \operatorname{deg}\left(B_{0}\right)<h-1$ and $\operatorname{deg}\left(C_{0}\right)<$ $h-1$. The result follows with $A(X)=(1-X) A_{0}(X), B(X)=(1-X) B_{0}(X)$ and $C(X)=$ $\left(1-X^{2}\right) C_{0}(X)$. 
Lemma 14 For all $h \geq 0$,

$$
\sum_{r=0}^{h-1} s(h, r) r=\frac{h}{2}(s(h)-2)
$$

Proof: Let $Z_{h}=\sum_{r=0}^{h} s(h, r) r$. Then

$$
\begin{array}{rlr}
Z_{h} & =\sum_{r=0}^{h} s(h, r) r & \\
& =\sum_{u=0}^{h} s(h, h-u)(h-u) & \text { by setting } r=h-u \\
& =\sum_{u=0}^{h} s(h, u)(h-u) & \\
& =h \sum_{u=0}^{h} s(h, u)-\sum_{u=0}^{h} s(h, u) u & \\
& =h s(h)-Z_{h} &
\end{array}
$$

Hence $Z_{h}=\frac{h}{2} s(h)$ and $\sum_{r=0}^{h-1} s(h, r) r=Z_{h}-h=\frac{h}{2}(s(h)-2)$.

Lemma 15 For all $h \geq 1$,

$$
\sum_{L=h}^{2 h-1} s(L, h)-\sum_{L=0}^{h-1} s(L, h)=s(h)+s(h-1)-(h+1)
$$


Proof:

$$
\begin{aligned}
\sum_{L=h}^{2 h-1} s(L, h)-\sum_{L=0}^{h-1} s(L, h) & \\
= & \sum_{L=0}^{h-1} s(L+h, h)-\sum_{L=0}^{h-1} s(L, h) \\
= & \sum_{L=0}^{h-1} s(L+h, L)-\sum_{L=0}^{h-1} s(L, h) \\
= & \sum_{L=0}^{h-1}(s(h, L)+s(h-1, L)-s(h-L-1, L)+s(L-1, L+h-1)+s(L-1, L+h-2))-\sum_{L=0}^{h-1} s(L, h) \\
= & \sum_{L=0}^{h-1}(s(h, L)+s(h-1, L)-s(h-1-L, h-1)+s(L-1, h)+s(L-1, h-1))-\sum_{L=0}^{h-1} s(L, h) \\
= & \sum_{L=0}^{h-1} s(h, L)+\sum_{L=0}^{h-1} s(h-1, L)-\sum_{L=0}^{h-1} s(h-1-L, h-1)+\sum_{L=0}^{h-1} s(L-1, h)+\sum_{L=0}^{h-1} s(L-1, h-1)-\sum_{L=0}^{h-1} s(L, h) \\
= & s(h)-1+s(h-1)-\left(\sum_{u=0}^{h-1} s(u, h-1)-\sum_{L=0}^{h-1} s(L-1, h-1)\right)-\left(\sum_{L=0}^{h-1} s(L, h)-\sum_{L=0}^{h-1} s(L-1, h)\right) \\
= & s(h)-1+s(h-1)-1-(h-1) \\
= & s(h)+s(h-1)-(h+1)
\end{aligned}
$$

We are now ready to prove the main theorem of this section. Proof of Theorem 12; We first prove existence. In Equation 1 we write

$$
\begin{aligned}
& R(X)=r_{0}+r_{1}(1-X)+r_{2}(1-X)^{2} \\
& A(X)=\sum_{k=0}^{h-2} a_{k} X^{k}, \quad B(X)=\sum_{k=0}^{h-1} b_{k} X^{k}, \quad C(X)=\sum_{k=0}^{h} c_{k} X^{k} .
\end{aligned}
$$

Thus

$$
\mathcal{S}_{h}(X)=\frac{r_{0}}{(1-X)^{3}}+\frac{r_{1}}{(1-X)^{2}}+\frac{r_{2}}{1-X}+\frac{A(X)}{1-X^{h-1}}+\frac{B(X)}{1-X^{h}}+\frac{C(X)}{1-X^{h+1}} .
$$


Since

$$
\frac{1}{(1-X)^{3}}=\sum_{L \geq 0} \frac{(L+1)(L+2)}{2} X^{L}, \quad \frac{1}{(1-X)^{2}}=\sum_{L \geq 0}(L+1) X^{L} \quad \text { and } \quad \frac{1}{1-X}=\sum_{L \geq 0} X^{L}
$$

the series expansion of $\mathcal{S}_{h}(X)$ is

$$
\begin{aligned}
\mathcal{S}_{h}(X)= & \sum_{L \geq 0} r_{0} \frac{(L+1)(L+2)}{2} X^{L}+\sum_{L \geq 0} r_{1}(L+1) X^{L}+\sum_{L \geq 0} r_{2} X^{L} \\
& +\sum_{n \geq 0} A(X) X^{n(h-1)}+\sum_{n \geq 0} B(X) X^{n h}+\sum_{n \geq 0} C(X) X^{n(h+1)} \\
= & \sum_{L \geq 0}\left(r_{0} \frac{(L+1)(L+2)}{2}+r_{1}(L+1)+r_{2}\right) X^{L} \\
& +\sum_{n \geq 0} \sum_{k=0}^{h-2} a_{k} X^{n(h-1)+k}+\sum_{n \geq 0} \sum_{k=0}^{h-1} b_{k} X^{n h+k}+\sum_{n \geq 0} \sum_{k=0}^{h} c_{k} X^{n(h+1)+k} \\
= & \sum_{L \geq 0}\left(\frac{r_{0}}{2} L^{2}+\left(\frac{3}{2} r_{0}+r_{1}\right) L+r_{0}+r_{1}+r_{2}+a_{L \bmod (h-1)}+b_{L} \bmod h+c_{L \bmod (h+1)}\right) X^{L}
\end{aligned}
$$

We get the result with $\alpha=\frac{1}{2} r_{0}, \beta=\frac{3}{2} r_{0}+r_{1}, u_{i}=a_{i}+r_{0}+r_{1}+r_{2}, v_{i}=b_{i}$ and $w_{i}=c_{i}$.

From the Taylor series of $(1-X)^{3} \mathcal{S}_{h}(X)$ at $X=1$, we get $r_{0}=\frac{\mathcal{F}_{h}(1)}{h\left(h^{2}-1\right)}$ and $r_{1}=$ $\frac{\frac{3}{2}(h-1) \mathcal{F}_{h}(1)-\mathcal{F}_{h}^{\prime}(1)}{h\left(h^{2}-1\right)}$. We have

$$
\mathcal{F}_{h}(1)=2 \sum_{r=0}^{h-2} s(h-1, r)=2(s(h-1)-1)
$$

and by Lemmas 14 and 15 .

$$
\begin{aligned}
\mathcal{F}_{h}^{\prime}(1) & =-(h-1)\left(\sum_{L=h}^{2 h-1} s(L, h)-\sum_{L=0}^{h-1} s(L, h)+(h-2)\right)+2 \sum_{r=0}^{h-2} s(h-1, r) r+(4 h-1) \sum_{r=0}^{h-2} s(h-1, r) \\
& =-(h-1)(s(h)+s(h-1)-(h+1)+(h-2))+2 \frac{h-1}{2}(s(h-1)-2)+(4 h-1)(s(h-1)-1) \\
& =-(h-1)(s(h)-1)+(4 h-1)(s(h-1)-1) .
\end{aligned}
$$

Thus we get finally

$$
\begin{aligned}
\alpha & =\frac{\mathcal{F}_{h}(1)}{2 h\left(h^{2}-1\right)}=\frac{s(h-1)-1}{h\left(h^{2}-1\right)}=\frac{1}{h\left(h^{2}-1\right)} \sum_{i=1}^{h-1}(h-i) \varphi(i) \\
\beta & =\frac{\frac{3}{2} h \mathcal{F}_{h}(1)-\mathcal{F}_{h}^{\prime}(1)}{h\left(h^{2}-1\right)}=\frac{s(h)-s(h-1)}{h(h+1)}=\frac{1}{h(h+1)} \sum_{i=1}^{h} \varphi(i) .
\end{aligned}
$$



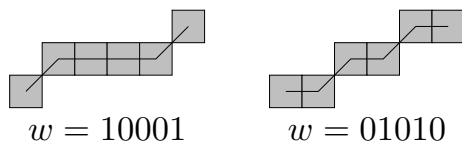

Figure 2: The two symmetrical segments of length 5 and height 2, and their respective encodings as balanced palindromes

\section{Balanced palindromes and symmetrical discrete segments}

In the present section, we focus on the case of segments which are symmetrical with respect to the point $(L / 2, h / 2)$. These segments are encoded by balanced palindromes.

\subsection{Recurrence formula}

This investigation is close to the general case by noticing that if $w$ is a palindrome, then so is $\theta_{0}(w)$. We first need an additional property of the mapping $\theta_{0}$.

Lemma 16 For all $w \in\{0,1\}^{*}$ and all $\alpha \geq 0$,

$$
\begin{aligned}
\theta_{0}(w 1) & =\theta_{0}(w) 1 \\
\theta_{0}\left(w 10^{\alpha+1}\right) & =\theta_{0}(w) 10^{\alpha}
\end{aligned}
$$

Proof: Easy induction on $|w|_{1}$.

Corollary 17 For all $w \in\{0,1\}^{*}, \theta_{0}\left(w^{\sim}\right)=\left(\theta_{0}(w)\right)^{\sim}$

Proof: By induction on $|w|_{1}$. If $|w|_{1}=0$ then $w=0^{\alpha}$ for some $\alpha \geq 0$ and the result obviously holds. Now assume that $|w|_{1} \geq 1$ and the result holds for all $v$ such that $|v|_{1}<|w|_{1}$. Then either $w=1 v$ and

$$
\begin{aligned}
& \theta_{0}\left(w^{\sim}\right)=\theta_{0}\left((1 v)^{\sim}\right) \\
& =\theta_{0}\left(v^{\sim} 1\right) \\
& =\theta_{0}\left(v^{\sim}\right) 1 \quad \text { by Lemma } 16 \\
& =\theta_{0}(v)^{\sim} 1 \quad \text { by the induction hypothesis } \\
& =\left(1 \theta_{0}(v)\right)^{\sim} \\
& =\theta_{0}(1 v)^{\sim} \quad \text { by definition of } \theta_{0} \\
& =\theta_{0}(w)^{\sim}
\end{aligned}
$$


or $w=0^{\alpha+1} 1 v$ and

$$
\begin{array}{rlrl}
\theta_{0}\left(w^{\sim}\right) & =\theta_{0}\left(\left(0^{\alpha+1} 1 v\right)^{\sim}\right) & \\
& =\theta_{0}\left(v^{\sim} 10^{\alpha+1}\right) & & \\
& =\theta_{0}\left(v^{\sim}\right) 10^{\alpha} & & \text { by Lemma 16 } \\
& =\theta_{0}(v)^{\sim} 10^{\alpha} & & \text { by the induction hypothesis } \\
& =\left(0^{\alpha} 1 \theta_{0}(v)\right)^{\sim} & & \\
& =\theta_{0}\left(0^{\alpha+1} 1 v\right)^{\sim} & & \text { by definition of } \theta_{0} \\
& =\theta_{0}(w)^{\sim} & &
\end{array}
$$

Corollary 18 If $w \in\{0,1\}^{*}$ is a palindrome, then $\theta_{0}(w)$ is also a palindrome.

Proof: Immediate consequence of Corollary 17

$\square$ We denote by $\mathbb{P}(L, h)$ the set of balanced palindromes of length $L$ and height $h$, and, for $x \in\{0,1\}$,

by $\mathbb{P}_{x}(L, h)$ the set of balanced palindromes of length $L$ and height $h$ the first (and last) letter of which is $x$. We define the function $p(L, h)$ on $\mathbb{Z}^{2}$ by:

$$
p(L, h)= \begin{cases}\# \mathbb{P}(L, h \bmod L) & \text { if } L>0 \\ 1 & \text { if } L=0 \text { and } h=0 \\ 0 & \text { if } L<0 \text { or } L=0 \text { and } h \neq 0\end{cases}
$$

and for $0 \leq h \leq L$ and $x \in\{0,1\}$, we define $p_{x}(L, h)=\# \mathbb{P}_{x}(L, h)$. We have the following properties.

Lemma 19 Let $L, h \in \mathbb{N}$ such that $0 \leq h \leq L$.

1. If $L \geq 2 h+1$, then $\theta_{0}$ is a bijection from $\mathbb{P}_{0}(L, h)$ to $\mathbb{P}(L-(h+1), h)$.

2. If $L \geq 2 h-1$, then $\theta_{0}$ is a bijection from $\mathbb{P}_{1}(L, h)$ to $\mathbb{P}_{1}(L-(h-1), h)$.

\section{Proof:}

1. Since $\mathbb{P}_{0}(L, h) \subset \mathbb{S}_{0,0}(L, h)$, from Lemma 6, we already know that $\theta_{0}\left(\mathbb{P}_{0}(L, h)\right) \subset \mathbb{S}(L-(h+$ $1), h)$ and from Corollary $18, \theta_{0}\left(\mathbb{P}_{0}(L, h)\right) \subset \mathbb{P}(L-(h+1), h)$. Since $\theta_{0}$ is injective on $\mathbb{S}_{0,0}(L, h)$, it is also injective on $\mathbb{P}_{0}(L, h)$. We are left to prove that it is surjective. Let $w \in \mathbb{P}(L-(h+1), h)$ and $w^{\prime}=\varphi_{0}(w) 0$. From Lemma 6, we have $w^{\prime} \in \mathbb{S}_{0,0}(L, h)$ and $\theta_{0}\left(w^{\prime}\right)=w$. We prove by induction on $|w|_{1}$ that $w^{\prime}$ is a palindrome. If $|w|_{1}=0$ then $w=0^{\alpha}$ for some $\alpha \geq 0$ and $w^{\prime}=0^{\alpha+1}$ which is trivially a palindrome. If $|w|_{1}=1$ then $w=0^{\alpha} 10^{\alpha}$ for some $\alpha \geq 0$ and $w^{\prime}=0^{\alpha+1} 10^{\alpha+1}$ which is again trivially a palindrome. If $|w|_{1} \geq 2$, assume that $\varphi_{0}(u) 0$ is a palindrome for all $u$ such that $|u|_{1}<|w|_{1}$. We have $w=0^{\alpha} 1 v 10^{\alpha}$ for some $\alpha \geq 0$ and some palindrome $v$ with $|v|_{1}<|w|_{1}$. Then $w^{\prime}=0^{\alpha+1} 1 \varphi_{0}(v) 010^{\alpha+1}$ is a palindrome because $\varphi_{0}(v) 0$ is a palindrome by the induction hypothesis.. 
2. The proof is similar. We get in the same way that $\theta_{0}\left(\mathbb{P}_{1}(L, h)\right) \subset \mathbb{P}_{1}(L-(h-1), h)$ and $\theta_{0}$ is injective on $\mathbb{P}_{1}(L, h)$. To prove that it is surjective, for each $w \in \mathbb{P}_{1}(L-(h-1), h)$ we consider $w^{\prime}$ such that $\varphi_{0}(w)=0 w^{\prime}$. From Lemma 6 we have $\theta_{0}\left(w^{\prime}\right)=w$ and $w^{\prime} \in \mathbb{S}_{1,1}(L, h)$. We prove like above that $w^{\prime}$ is a palindrome so that $w^{\prime} \in \mathbb{P}_{1}(L, h)$.

Lemma 20 For all $L, h \in \mathbb{N}$ such that $0 \leq h \leq L, p_{0}(L, h)=p(L-h-1, h)$ and $p_{1}(L, h)=$ $p(h-1, L-1)$

Proof: Similar to the proof of Lemma 8

$\square$ From Lemma 20 and the definition of $p(L, h)$, we deduce the following recurrence for $p(L, h)$.

Theorem 21 Let $L, h \in \mathbb{Z}$,

$$
p(L, h)= \begin{cases}0 & \text { if } L<0 \text { or }(L=0 \text { and } h \neq 0) \\ 1 & \text { if } L \geq 0 \text { and }(h=0 \text { or } h=L) \\ p(L, h \bmod L) & \text { if } L>0 \text { and }(h<0 \text { or } h>L) \\ p(L-h-1, h)+p(h-1, L-1) & \text { otherwise }\end{cases}
$$

Sample values of $p(L, h)$ are given in Table 3 .

\begin{tabular}{r||ccccccccccc}
$L \backslash h$ & 0 & 1 & 2 & 3 & 4 & 5 & 6 & 7 & 8 & 9 & 10 \\
\hline 0 & 1 & & & & & & & & & & \\
1 & 1 & 1 & & & & & & & & \\
2 & 1 & 0 & 1 & & & & & & & \\
3 & 1 & 1 & 1 & 1 & & & & & & \\
4 & 1 & 0 & 2 & 0 & 1 & & & & & & \\
5 & 1 & 1 & 2 & 2 & 1 & 1 & & & & \\
6 & 1 & 0 & 2 & 0 & 2 & 0 & 1 & & & & \\
7 & 1 & 1 & 3 & 2 & 2 & 3 & 1 & 1 & & & \\
8 & 1 & 0 & 3 & 0 & 2 & 0 & 3 & 0 & 1 & & \\
9 & 1 & 1 & 3 & 3 & 2 & 2 & 3 & 3 & 1 & 1 & \\
10 & 1 & 0 & 4 & 0 & 2 & 0 & 2 & 0 & 4 & 0 & 1
\end{tabular}

Table 3: Sample values of $p(L, h)$ for $0 \leq h \leq L \leq 10$

\subsection{Generating functions of $p(L, h)$}

In the same way we obtained generating functions for $s(L, h)$, we deduce generating functions for $p(L, h)$ from the recurrence above. We consider the generating functions $\mathcal{P}_{h}(X)=\sum_{L \geq 0} p(L, h) X^{L}$ 
Theorem 22 One has: $\mathcal{P}_{0}(X)=\frac{1}{1-X}, \mathcal{P}_{1}(X)=\frac{X}{1-X^{2}}$ and for all $h \geq 2$,

$$
\mathcal{P}_{h}(X)=\frac{1}{1-X^{h+1}}\left(\sum_{L=0}^{h-1} p(L, h) X^{L}+\frac{X^{h}}{1-X^{h-1}} \sum_{r=0}^{h-2} p(h-1, r) X^{r}\right)
$$

Proof: One has:

$$
\begin{aligned}
\mathcal{P}_{0}(X) & =\sum_{L \geq 0} p(L, 0) X^{L}=\sum_{L \geq 0} X^{L}=\frac{1}{1-X} \\
\mathcal{P}_{1}(X) & =\sum_{L \geq 0} p(L, 1) X^{L} \\
& =p(0,1)+p(1,1) X+\sum_{L \geq 2} p(L, 1) X^{L} \\
& =X+\sum_{L \geq 2}(p(L-2,1)+p(0, L-1)) X^{L} \\
& =X+X^{2} \sum_{L \geq 0} p(L, 1) X^{L} \\
& =X+X^{2} \mathcal{P}_{1}(X)
\end{aligned}
$$

Hence, $\mathcal{P}_{1}(X)=\frac{X}{1-X^{2}}$. 
For all $h \geq 2$, we have

$$
\begin{aligned}
\mathcal{P}_{h}(X) & =\sum_{L \geq 0} p(L, h) X^{L} \\
& =\sum_{L=0}^{h-1} p(L, h) X^{L}+\sum_{L \geq h} p(L, h) X^{L} \\
& =\sum_{L=0}^{h-1} p(L, h) X^{L}+X^{h} \sum_{L \geq 0} p(L+h, h) X^{L} \\
& =\sum_{L=0}^{h-1} p(L, h) X^{L}+X^{h} \sum_{L \geq 0}(p(L-1, h)+p(h-1, L+h-1)) X^{L} \\
& =\sum_{L=0}^{h-1} p(L, h) X^{L}+X^{h} \sum_{L \geq 0} p(L-1, h) X^{L}+X^{h} \sum_{L \geq 0} p(h-1, L) X^{L} \\
& =\sum_{L=0}^{h-1} p(L, h) X^{L}+X^{h+1} \mathcal{P}_{h}(X)+X^{h} \sum_{q \geq 0} \sum_{r=0}^{h-2} p(h-1, q(h-1)+r) X^{q(h-1)+r} \\
& =\sum_{L=0}^{h-1} p(L, h) X^{L}+X^{h+1} \mathcal{P}_{h}(X)+X^{h} \sum_{q \geq 0} X^{q(h-1)} \sum_{r=0}^{h-2} p(h-1, r) X^{r} \\
& =X^{h+1} \mathcal{P}_{h}(X)+\sum_{L=0}^{h-1} p(L, h) X^{L}+\frac{X^{h}}{1-X^{h-1}} \sum_{r=0}^{h-2} p(h-1, r) X^{r}
\end{aligned}
$$

Finally, we get

$$
\begin{aligned}
\mathcal{P}_{h}(X) & =\frac{1}{1-X^{h+1}}\left(\sum_{L=0}^{h-1} p(L, h) X^{L}+\frac{X^{h}}{1-X^{h-1}} \sum_{r=0}^{h-2} p(h-1, r) X^{r}\right) \\
& =\frac{\left(1-X^{h-1}\right) \sum_{L=0}^{h-1} p(L, h) X^{L}+X^{h} \sum_{r=0}^{h-2} p(h-1, r) X^{r}}{\left(1-X^{h-1}\right)\left(1-X^{h+1}\right)} \\
& =\frac{\mathcal{G}_{h}(X)}{\left(1-X^{h-1}\right)\left(1-X^{h+1}\right)}
\end{aligned}
$$

where $\mathcal{G}_{h}(X) \in \mathbb{Z}[X]$ and $\operatorname{deg}\left(\mathcal{G}_{h}\right) \leq 2 h-2$.

$\square$ Sample values of $\mathcal{P}_{h}(X)$ are given in Table 4

\subsection{Asymptotic behaviour of $p(L, h)$}

As before, from the generating function, we deduce an expression of $p(L, h)$ which highlights its asymptotic behaviour. We prove the following theorem. 
On the Number of Balanced Words of Given Length and Height over a Two-Letter Alphabet

$$
\begin{aligned}
& \mathcal{P}_{2}(X)=\frac{X}{(1-X)\left(1-X^{3}\right)} \quad \mathcal{P}_{3}(X)=\frac{X}{\left(1-X^{2}\right)\left(1-X^{4}\right)} \\
& \mathcal{P}_{4}(X)=\frac{X+X^{2}+X^{3}}{\left(1-X^{3}\right)\left(1-X^{5}\right)} \quad \mathcal{P}_{5}(X)=\frac{X+X^{3}+X^{7}}{\left(1-X^{4}\right)\left(1-X^{6}\right)} \\
& \mathcal{P}_{6}(X)=\frac{X+X^{2}+X^{3}+2 X^{4}+X^{5}+X^{8}}{\left(1-X^{5}\right)\left(1-X^{7}\right)}
\end{aligned}
$$

Table 4: Sample values of $\mathcal{P}_{h}(X)$

Theorem 23 For all $h \geq 2$ there exist $u_{0}, \ldots, u_{h-2}, v_{0}, \ldots, v_{h} \in \mathbb{Q}$ such that:

- if $h$ is even then

$$
\forall L \geq 0, \quad p(L, h)=\alpha L+u_{L \bmod (h-1)}+v_{L \bmod (h+1)}
$$

- if $h$ is odd then

$$
\forall L \geq 0, \quad p(L, h)=\alpha\left(1-(-1)^{L}\right) L+u_{L \bmod (h-1)}+v_{L \bmod (h+1)}
$$

where $\alpha=\frac{1}{h^{2}-1} \sum_{i=1}^{\left\lceil\frac{h-1}{2}\right\rceil} \varphi(h+1-2 i)$

Before proving this theorem, we need some lemmas.

Lemma 24 For all $h \geq 2$ :

- if $h$ is even then there exist $R, A, B \in \mathbb{Q}[X]$ such that $\operatorname{deg}(R)<2, \operatorname{deg}(A)<h-1, \operatorname{deg}(B)<h+1$ and

$$
\mathcal{P}_{h}(X)=\frac{R(X)}{(1-X)^{2}}+\frac{A(X)}{1-X^{h-1}}+\frac{B(X)}{1-X^{h+1}}
$$

- if $h$ is odd then there exist $Q, R, A, B \in \mathbb{Q}[X]$ such that $\operatorname{deg}(Q)<2, \operatorname{deg}(R)<2, \operatorname{deg}(A)<h-1$, $\operatorname{deg}(B)<h+1$ and

$$
\mathcal{P}_{h}(X)=\frac{Q(X)}{(1+X)^{2}}+\frac{R(X)}{(1-X)^{2}}+\frac{A(X)}{1-X^{h-1}}+\frac{B(X)}{1-X^{h+1}}
$$

Proof: If $h$ is even then $\mathcal{P}_{h}(X)$ may be written as

$$
\mathcal{P}_{h}(X)=\frac{\mathcal{G}_{h}(X)}{(1-X)^{2} \frac{1-X^{h-1}}{1-X} \frac{1-X^{h+1}}{1-X}} .
$$


Since $(1-X)^{2}, \frac{1-X^{h-1}}{1-X}$ and $\frac{1-X^{h+1}}{1-X}$ are pairwise coprime, there exist $R, A_{0}, B_{0} \in \mathbb{Q}[X]$ such that $\operatorname{deg}(R)<2, \operatorname{deg}\left(A_{0}\right)<h-2, \operatorname{deg}\left(B_{0}\right)<h$ and

$$
\mathcal{P}_{h}(X)=\frac{R(X)}{(1-X)^{2}}+\frac{A_{0}(X)}{\frac{1-X^{h-1}}{1-X}}+\frac{B_{0}(X)}{\frac{1-X^{h+1}}{1-X}}
$$

We get the result with $A(X)=(1-X) A_{0}(X)$ and $B(X)=(1-X) B_{0}(X)$.

If $h$ is odd then $1-X^{h-1}$ and $1-X^{h+1}$ are also dividable by $1+X$ so that we may write

$$
\mathcal{P}_{h}(X)=\frac{\mathcal{G}_{h}(X)}{(1+X)^{2}(1-X)^{2} \frac{1-X^{h-1}}{1-X^{2}} \frac{1-X^{h+1}}{1-X^{2}}} .
$$

Since $(1+X)^{2},(1-X)^{2}, \frac{1-X^{h-1}}{1-X^{2}}$ and $\frac{1-X^{h+1}}{1-X^{2}}$ are pairwise coprime, there exist $Q, R, A_{0}, B_{0} \in$ $\mathbb{Q}[X]$ such that $\operatorname{deg}(Q)<2, \operatorname{deg}(R)<2, \operatorname{deg}\left(A_{0}\right)<h-3, \operatorname{deg}\left(B_{0}\right)<h-1$ and

$$
\mathcal{P}_{h}(X)=\frac{Q(X)}{(1+X)^{2}}+\frac{R(X)}{(1-X)^{2}}+\frac{A_{0}(X)}{\frac{1-X^{h-1}}{1-X^{2}}}+\frac{B_{0}(X)}{\frac{1-X^{h+1}}{1-X^{2}}}
$$

We get the result with $A(X)=\left(1-X^{2}\right) A_{0}(X)$ and $B(X)=\left(1-X^{2}\right) B_{0}(X)$.

Lemma 25 For all $L, h \in \mathbb{N}$ such that $L$ is even and $h$ is odd, $p(L, h)=0$.

Proof: By definition of $p(L, h)$, the result is obvious if $L=0$. Also, it is sufficient to prove the result for $0 \leq h \leq L$ because $h$ and $h \bmod L$ have the same parity if $L$ is even. In this case, $p(L, h)$ is exactly the number of balanced palindromes of length $L$ and height $h$. Let $w$ be a palindrome of length $L$. If $L$ is even then $w=u u^{\sim}$ for some $u \in\{0,1\}^{*}$ and $|w|_{1}=2|u|_{1}$. Hence, there exist no palindrome of even length and odd height.

$\square$ We are now ready to prove the main theorem of this section. Proof of Theorem 23;

The proof is similar to the proof of Theorem 12. In Equations 2 and 3 , we write

$$
\begin{aligned}
& R(X)=\alpha+\beta(1-X), \quad Q(X)=\alpha^{\prime}+\beta^{\prime}(1+X) \\
& A(X)=\sum_{k=0}^{h-2} a_{k} X^{k}, \quad B(X)=\sum_{k=0}^{h} b_{k} X^{k}
\end{aligned}
$$

and we get for all $h \geq 2$ : 
- if $h$ is even then

$$
\mathcal{P}_{h}(X)=\frac{\alpha}{(1-X)^{2}}+\frac{\beta}{1-X}+\frac{A(X)}{1-X^{h-1}}+\frac{B(X)}{1-X^{h+1}}
$$

and the series expansion of $\mathcal{P}_{h}(X)$ is

$$
\begin{aligned}
\mathcal{P}_{h}(X) & =\sum_{L \geq 0}(\alpha(L+1)+\beta) X^{L}+\sum_{n \geq 0} A(X) X^{n(h-1)}+\sum_{n \geq 0} B(X) X^{n(h+1)} \\
& =\sum_{L \geq 0}(\alpha(L+1)+\beta) X^{L}+\sum_{n \geq 0} \sum_{k=0}^{h-2} a_{k} X^{n(h-1)+k}+\sum_{n \geq 0} \sum_{k=0}^{h} b_{k} X^{n(h+1)+k} \\
& =\sum_{L \geq 0}\left(\alpha L+\alpha+\beta+a_{L \bmod (h-1)}+b_{L \bmod (h+1)}\right) X^{L}
\end{aligned}
$$

Hence

$$
\forall L \geq 0, p(L, h)=\alpha L+\alpha+\beta+a_{L \bmod (h-1)}+b_{L \bmod (h+1)}
$$

- if $h$ is odd then

$$
\mathcal{P}_{h}(X)=\frac{\alpha^{\prime}}{(1+X)^{2}}+\frac{\beta^{\prime}}{1+X}+\frac{\alpha}{(1-X)^{2}}+\frac{\beta}{1-X}+\frac{A(X)}{1-X^{h-1}}+\frac{B(X)}{1-X^{h+1}}
$$

and its series expansion is

$$
\mathcal{P}_{h}(X)=\sum_{L \geq 0}\left(\alpha^{\prime}(-1)^{L}(L+1)+\beta^{\prime}(-1)^{L}+\alpha(L+1)+\beta+a_{L \bmod (h-1)}+b_{L \bmod (h+1)}\right) X^{L}
$$

Hence

$\forall L \geq 0, p(L, h)=\alpha^{\prime}(-1)^{L}(L+1)+\beta^{\prime}(-1)^{L}+\alpha(L+1)+\beta+a_{L \bmod (h-1)}+b_{L \bmod (h+1)}$

Considering the Taylor expansion of $(1-X)^{2} \mathcal{P}_{h}(X)$ at $X=1$ and, in case $h$ is even, $(1+X)^{2} \mathcal{P}_{h}(X)$ at $X=-1$, we get

$$
\alpha=\frac{\mathcal{G}_{h}(1)}{h^{2}-1}=\frac{1}{h^{2}-1} \sum_{r=0}^{h-2} p(h-1, r)=\frac{1}{h^{2}-1} \sum_{i=1}^{\left\lceil\frac{h-1}{2}\right\rceil} \varphi(h+1-2 i)
$$

and, if $h$ is odd,

$$
\alpha^{\prime}=\frac{\mathcal{G}_{h}(-1)}{h^{2}-1}=\frac{(-1)^{h}}{h^{2}-1} \sum_{r=0}^{h-2}(-1)^{r} p(h-1, r)=\frac{-1}{h^{2}-1} \sum_{r=0}^{h-2} p(h-1, r)
$$

where the last equality is deduced from Lemma25. Hence $\alpha^{\prime}=-\alpha$.

Finally, we get the result with $u_{i}=\alpha+\beta+a_{i}$ if $h$ is even and $u_{i}=\left(1-(-1)^{i}\right) \alpha+\beta+(-1)^{i} \beta^{\prime}+a_{i}$ if $h$ is odd and $v_{i}=b_{i}$.

Acknowledgements: The authors would like to thank Julien Cassaigne for useful discussions and comments. 


\section{References}

[BL88] C. A. Berenstein and D. Lavine. On the number of digital straight line segments. P.A.M.I, 10(6):880-887, 1988.

[BP93] J. Berstel and M. Pocchiola. A geometric proof of the enumeration formula for Sturmian words. Int. J. Algebra Comput., 3(3):349-355, 1993.

[Chr75] E. B. Christoffel. Observatio arithmetica. Math. Ann., 6:145-152, 1875.

[CHT02] J. Cassaigne, P. Hubert, and S. Troubetzkoy. Complexity and growth for polygonal billiards. Annales de l'Institut Fourier, 52:1001-1013, 2002. 12 pages, 4 figures.

[CM91] J.-M. Chassery and A. Montanvert. Géométrie discrète en analyse d'images. Hermès, Paris, 1991.

[dLdL05] Aldo de Luca and Alessandro de Luca. Palindromes in sturmian words. In Clelia de Felice and Antonio Restivo, editors, Developments in Language Theory, volume 3572 of Lecture Notes in Computer Science, pages 199-208. Springer, 2005.

[Fre74] H. Freeman. Computer processing of line-drawing images. ACM Computing Surveys, 6(1):5797, 1974.

[Hun85] S. H. Y. Hung. On the straightness of digital arcs. In IEEE Transactions on Pattern Analysis and Machine Intelligence, volume PAMI-7, pages 203-215, 1985.

[Lot02] Lothaire. Algebraic Combinatorics On Words. Cambridge University Press, 2002.

[Mar82] A. Markoff. Sur une question de Jean Bernoulli. Math. Ann., 19:27-36, 1882.

[MH40] M. Morse and G. A. Hedlund. Symbolic dynamics II. Sturmian trajectories. Amer J. Math., 62:1-42, 1940 .

[Mig91] F. Mignosi. On the number of factors of sturmian words. Theoret. Comput. Sci., 82(1):71-84, 1991.

[Par97] Bruno Parvaix. Propriétés d'invariance des mots sturmiens. J. Théor. Nombres Bordeaux, 9:351-369, 1997.

[Rev91] J.-P. Reveillès. Géométrie discrète, calcul en nombres entiers et algorithmique. Thèse d'état, Université Louis Pasteur, Strasbourg, 1991.

[Ros74] A. Rosenfeld. Digital straight lines segments. In IEEE Transactions on Computers, pages 1264-1369, 1974. 